\title{
Interleukin-19 protects mice from oxazolone-induced colonic inflammation
}

\author{
Mariko Miki, Yasuyuki Fujimoto, Hidemitsu Nakajima, Tadayoshi Takeuchi, Yasu-Taka Azuma
}

Laboratory of Veterinary Pharmacology, Division of Veterinary Science, Osaka Prefecture University Graduate School of Life and Environmental Science, Japan

Background: IL-19 was originally found by sequence homology to IL-10, and is a member of the IL-10 family. However, little is known about the exact immunological role of IL-19 in the regulation of inflammatory bowel disease (IBD) that is characterized by dysregulated intestinal inflammation and mucosal tissue damage in parts of the gastrointestinal tract. Clinically, ulcerative colitis (UC) and Crohns disease are two of the most common types of IBDs. UC involves the superficial mucosal and submucosal layers of the colon and is driven by Th2 cytokines, such as IL-4, IL-5, and IL-13. In this study, we investigated the role of IL-19 in oxazolone-induced colitis which is useful for the study of the Th2 inflammatory response and resembles the symptom seen in UC.

Methods: Balb/c genetic background IL-19 knockout (KO) mice were used. To presensitize mice, $150 \mu \mathrm{L}$ of a 4\% solution of oxazolone in $100 \%$ ethanol was applied in the shaved abdominal skin. Seven days after presensitization, colitis was induced by intrarectal administration with $100 \mu \mathrm{L}$ of $3 \%$ of oxazolone in $50 \%$ ethanol using a plastic catheter. The colitis was evaluated by analyzing body weight loss and histology of the distal colon.

Results: IL-19 KO mice showed severe weight loss compared with wild-type (WT) mice from day 1 to day 2. Histological analysis revealed that the distal colon in IL-19 KO mice exhibited increased numbers of infiltrating cells and a general loss of tissue architecture. In IL-19 KO mice, oxazolone treatment increased colonic MPO activity. Serum IgE levels and the number of circulating eosinophil were significantly elevated in IL-19 KO mice in comparison with WT mice. The exacerbation of oxazolone-induced colonic inflammation following IL-19 knockout was accompanied by increased production of IL-4. CD4-positive T-cells isolated from lymph node of IL-19 KO mice with oxazolone-induced colitis produced elevated amounts of IL-2, IL-4, IL-9, and CCL5, but not IL-5 and IL-13, upon stimulation with anti-CD3 and anti-CD28 antibodies in vitro.

Conclusions: IL-19 plays an anti-inflammatory role in the Th2-mediated colitis model, suggesting that IL-19 may represent potential therapeutic target for reducing colonic inflammation. 